\title{
LA Colombie DONT LE MONDE A BESOIN : À PROPOS DES DIALOGUES DE PAIX
}

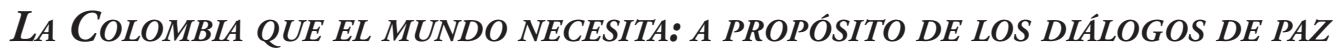

\section{Richard Petris ${ }^{1}$}

\section{Resume}

L'Ecole de la paix travaille depuis plus de 20 ans avec différents secteurs de la société colombienne (ONG, institutions, militaires, communautés), afin d'œuvrer au rapprochement pour le vivre ensemble, par l'analyse, le dialogue et la construction commune d'alternatives à la violence, par la méthode de "l'approche globale».

Fort de son expérience en France, en Colombie, mais aussi dans d'autres pays qu'il a parcourus (Cambodge, Brésil, Rwanda, Côte d'Ivoire, Ex-Yougoslavie), Richard Petris propose dans cet article de lire les attentes nées du processus de paix de La Havane sous un angle historique et international qui rappelle combien la Colombie doit être un exemple de réconciliation.

Mot cles: approche globale, culture de la paix, international, militaires, réconciliation.

\section{Resuimen}

La Escuela de la paz lleva más de 20 años trabajando con distintos sectores de la sociedad colombiana (ONG, instituciones militares, comunidades) con el fin de obrar al acercamiento para la convivencia, desde el análisis, el dialogo y la construcción común de alternativas a la violencia, con el "método global".

Con su experiencia en Francia, Colombia, pero también en otros países que ha recorrido (Camboya, Brasil, Ruanda, Costa de Marfil, Ex Yugoslavia), Richard Petris propone, en este articulo, leer las expectativas generadas por el proceso de paz de La Habana bajo una lectura histórica e internacionalista, que nos recuerde hasta que punto Colombia debe ser un ejemplo de reconciliación.

Palabras clave: enfoque global, cultura de la paz, internacional, militares, reconciliación.

Recibido: 15 de noviembre de 2015 Aceptado: 05 de diciembre de 2015

L'Histoire va-t-elle hésiter encore longtemps en Colombie ? Ceci n'est qu'une manière un peu moins directe, un peu moins abrupte - "mas suave " dirons-nous ! - de nous adresser, en réalité, à nos amis Colombiens pour leur dire avec quel espoir nous guettons le signal qui viendra de La Havane pour nous confirmer la nouvelle que nous attendons depuis si longtemps de la signature d'un accord mettant fin aux combats et engageant effectivement le pays dans le post-conflit et la rude tâche de la construction d'une paix véritable et durable. Notre impatience n'est pas feinte et, en tant qu'Ecole de la paix, nous nous sentons totalement solidaires de tous les acteurs de ces négociations qui semblent avoir fait le

1 Institution: Ecole de la paix de Grenoble - France. Richard Petris est diplômé de l'Institut d'Etudes Politiques de Grenoble. Fondateur, ex-directeur (1989-2012) et membre du Conseil d'Administration (depuis 2012) de l'Ecole de la paix, organisation qui promeut la culture de la paix par l'éducation, du niveau local à international. Editions Charles Léopold Mayer, Paris, 2016. Correo: richard.petris@ecoledelapaix.org 
choix d'aller jusqu'à leur terme positif, en en acceptant la durée. Comment expliquer notre impatience et quel intérêt peut-on invoquer, quels bénéfices estimons-nous qu'en tireront non seulement toute la population colombienne mais également la communauté internationale ?

\section{Un but, un plan, une méthode...!}

Cela fait presque 25ans que nous avions cru percevoir une lueur : au début des années 90, les effectifs de la guérilla, des FARC en particulier, atteignaient un creux que de bons connaisseurs colombiens de la situation du pays considéraient comme un signe qui devait les inciter à tirer les leçons de ce déclin et du changement de l'environnement international avec la fin de la guerre froide pour décider de retourner à la légalité. Nous étions alors trois organisations françaises, ou représentant la société civile en France, dans le cadre d'un séminaire organisé par le Cinep à Bogota pour penser la résolution du conflit interne au pays, à la lumière, notamment, de ce qu'on pouvait tirer de la démarche qui était en cours au Salvador et qui conduisait l'Organisation des Nations Unies à mettre en œuvre les accords de paix effectivement ambitieux qui venaient d'être signés. Nous avions été frappés par des souhaits qui s'étaient exprimés au cours de cette rencontre : "Ah ! Si la communauté internationale pouvait s'occuper de nous comme elle s'occupe du Salvador! »

Nous prenions conscience alors que, tout en n'étant pas de même intensité, les deux situations pouvaient être scrutées en profondeur de la même manière pour mesurer à quel point ces conflits internes avaient des racines profondes et des causes structurelles qui ne pouvaient être négligées dans le cadre d'une recherche de solution. Mais, dans quelque situation de conflit que ce soit, peut-on, en réalité, ignorer cette constante? Manifestement, dans le cas colombien, l'une des explications fortes du regain de forme de la guérilla dans les années qui suivirent, se situait dans le développement du recours par celle-ci aux bénéfices de la drogue. Il n'en demeure pas moins que, comme nous l'affirma au début des années 2000 un officier chargé des opérations d'une Brigade de l'armée colombienne dans l'une des régions les plus affectées par la violence, rien ne serait jamais réglé dans ce pays « tant que la question de la terre ne serait pas résolue ! »Cette question ne pouvant certainement pas être déconnectée non plus du problème qui nous est apparu bien ancien que ce pays devait décidément avoir avec « le pouvoir » dans toutes les sphères de la société et à ses différents niveaux.

Si la Colombie se distingue par la profusion des richesses issues de son sol, qu'elles soient minières ou agricoles, ses habitants ne sont pas en reste et démontrent une qualité humaine, en même temps que de son personnel, qui explique certainement la capacité de résilience de cette nation. En dépit de son caractère d' "Etat inachevée " comme le décrivent certains et bien que dotée d'une constitution reconnue pourtant comme plutôt moderne par plus d'une de ses dispositions faisant, notamment, davantage place à la participation populaire, ses atouts sont réels. C'est dans ce contexte mitigé que le lancement d'une "Asamblea permanente de la sociedad civil por la paz » réunit, en 1998, plus de 3000 représentants d'une multitude de mouvements et d'organisations colombiennes, accompagnés de près d'une centaine de représentants internationaux et se réclamant ensemble, à la fois, des droits humains fondamentaux et d'une conception plus globale de la construction de la paix. Il était évident que, sur le moment, l'écho de cet appel à une initiative sérieuse pour la paix n'atteindrait qu’à peine les sphères $\mathrm{du}$ pouvoir politique, économique et social et ne pouvait donc être de nature à changer la donne.

Cependant, en un raccourci nécessairement rapide, on peut certainement voir dans l'initiative de paix qui a été prise par le gouvernement du président Santos, il y aura bientôt quatre ans, une réponse à la demande qui ne faisait qu'exprimer la seule prise de position collective se voulant constructive et porteuse d'avenir. C'est la raison pour laquelle, vue de l'extérieur, la décision claire de ce gouvernement nous est apparue comme susceptible de faire avancer un véritable processus de recherche d'un accord, 
parce que choisissant, comme en d'autres circonstances et pour engager une véritable stratégie - par définition donc, ainsi qu'ont pu l'énoncer des chefs militaires ayant notamment contribué à hâter la fin de la première guerre mondiale dont on marque le centenaire actuellement - une règle d'action condition d'efficacité : " un but, un plan, une méthode... et agir avec détermination et opiniâtreté. " Pour reprendre une démarche plus détachée de la chose militaire et dont nous estimons à l'Ecole de la paix qu'elle peut être la garantie du succès en matière de construction de la paix, nous avons eu le sentiment que la méthode choisie conduirait bien, cette fois, à appliquer les trois temps nécessaires : la rencontre qui seule peut permettre le dialogue, la négociation qui seule prépare le compromis et la réconciliation qui seule peut conduire au vivre-ensemble.

\section{Un bénéfice de portée universelle}

Il nous est arrivé, dans un des points les plus reculés de Colombie de faire rêver certains de nos interlocuteurs, parfois pris entre deux feux et souffrant généralement d'une menace quasi quotidienne $\mathrm{du}$ fait de ce conflit interne, en leur expliquant ce que nos pays européens, au sortir de la seconde guerre mondiale, avaient décidé d'entreprendre à l'instigation d'hommes politiques sages et avisés, pour mettre fin à des guerres inexpugnables et faire une réalité de l'affirmation : "Plus jamais ça! ». Car nous y sommes parvenus en réalisant une expérience unique dans l'histoire de l'humanité, en faisant de cette entreprise de dialogue, puis de construction commune conduisant à une réconciliation, que certains estiment, à bon droit sans doute, irréversible, une véritable " aventure de paix " par l'application de cette méthode. Et c'est d'ailleurs ce que nous sommes allés exposer également à l'Ecole supérieure de guerre de Bogota, à l'occasion d'une rencontre organisée à l'occasion d'un échange avec l'Ecole de guerre française en 2011. Notre propos consistait en effet, dans cette collaboration avec les forces de sécurité de nos deux pays, au niveau d'une réflexion intellectuelle et d'ateliers de travail prenant en considération différents cas et différentes expériences sur plusieurs terrains et continents, à faire ressortir la nécessité d'une " approche globale » pour construire la paix.

Lorsque nous parlons d' "approche globale» nous dépassons, en effet, la seule dimension militaire, prenant en compte les conditions plurielles de la paix et du vivre-ensemble. Nous ne pouvions, dès lors, qu'adhérer à la définition des cinq grands thèmes, telles la question agraire, la justice, la participation à la vie politique, la question de la drogue, devant faire l'objet d'une négociation que l'on continuerait jusqu'à ce qu' elle aboutisse. En recherchant et trouvant ainsi un accord sur chacun des points de l'agenda des négociations, les dialogues de paix de La Havane ne répondent pas qu'aux seules préoccupations colombiennes. De même qu'ils sont l'occasion de solliciter l'expertise qui s'est constituée dans d'autres situations, en matière de justice transitionnelle par exemple ou de participation à la vie politique, ils contribuent à un échange d'expérience d'autant plus opportun qu'en dépit d'événements qui résultent de contentieux toujours non résolus, au Moyen-Orient par exemple, voire de nouveaux motifs de tension résultant des conséquences déstabilisatrices de la mondialisation, les avancées colombiennes viendront s'ajouter à une capitalisation générale, mondiale, utile sur les processus de paix.

En effet, les dialogues de paix en cours participent, selon nous, de deux mouvements nouveaux et profonds, qui, s'ils resteront évidemment contredits et toujours menacés par des retours en arrière dont les événements du Moyen-Orient mais aussi de nouvelles tensions internationales continuent de nous montrer le danger, méritent d'être davantage soulignés. Il s'agit d'abord de ce qu'un de nos amis, le général Jean Cot, évoque avec beaucoup d'espoir dans son dernier essai "Un monde en paix » (*) et qui est la formation progressive d'une " conscience universelle » dont il estime que " la manifestation la plus visible est sans doute l'émergence, d'un bout à l'autre du monde, d'un sentiment de révolte des opinions publiques, de la société civile face à des situations et des comportements inacceptables ". Mais il s'agit aussi de ce progrès de la culture de la paix auquel auront contribué ces négociations, comme nous 
considérons qu'il est également possible d'en repérer les signes d'en d'autres circonstances auxquelles nous nous intéressons. C'est le cas du Cambodge se relevant d'une autre tragédie et où l'on affirme publiquement la nécessité de développer une éducation à la paix pour promouvoir une telle culture dans le prolongement d'un tribunal pénal international qui, surmontant des critiques, montre la voie. Mais c'est aussi en Afghanistan, face à une autre complexité, où des hommes courageux ont entrepris d'héberger ensemble des étudiants de groupes ethniques ou de clans différents afin de préparer l'avenir du vivre-ensemble dans ce pays. Nous avons bien là ce que l'on doit considérer comme un début de réponse à la question que posait, Ilya Prigogine - prix Nobel de Chimie, lui - avant de disparaître, au début de ce millénaire : "Le plus important est de savoir comment passer politiquement d'une culture de guerre à une culture de paix ».

La Colombie n'est pas isolée. Allons jusqu’à reprendre à notre compte la question que posaient une organisation de la société civile nord-américaine au début de ces négociations : "La communauté internationale est-elle prête face à une Colombie du post-conflit ? " car ce qui est en train de se passer en Colombie, plus exactement à La Havane pour la Colombie, croyons-nous, est bien historique pour le pays et pour le monde. Tous les points traités sont constitutifs du projet national que le pays doit se donner, au-delà de la fin des combats, ce qui lui permettra de consolider son développement économique et social. Elle fera ainsi partie, sachons le reconnaître et en reprenant une des formules du général Cot déjà nommé, de « ceux qui marchent devant " et c'est bien de cette Colombie là que le monde a besoin. 